\title{
Kinetics of Molecular Recognition Mediated Nanoparticle Self-Assembly
}

\author{
Chinmay Soman ${ }^{1}$ and Todd Giorgio ${ }^{2}(\square)$ \\ ${ }^{1}$ Interdisciplinary Program in Materials Science, Vanderbilt University, 201 West End Avenue, Nashville, TN 37240, USA \\ ${ }^{2}$ Department of Biomedical Engineering, Vanderbilt University, 201 West End Avenue, Nashville, TN 37240, USA \\ Received: 14 September 2008 / Revised: 11 November 2008 / Accepted: 23 November 2008 \\ (C) Tsinghua University Press and Springer-Verlag 2009. This article is published with open access at Springerlink.com
}

\begin{abstract}
Nanoscale quantum dot-antibody conjugates have been shown to self-assemble to form micron-scale aggregates in the presence of specific proteomic antigen. The self-assembly process exhibits sigmoidal kinetics, suggesting that nucleation limits aggregation. Self-assembly kinetics in this study is characterized by flow cytometric analysis of the aggregation reaction over time. A range of physiologically relevant concentrations of the protein angiopoietin-2, a candidate cancer biomarker, are incubated with quantum dots conjugated with a polyclonal mixture of anti-angiopoietin- 2 antibodies. Antigen concentration modulates the slopes and inflection times of the sigmoidal kinetics curves. An understanding of self-assembly kinetics in this system may lead to improvements in sensitivity and specificity of this novel proteomic biomarker detection technique and improve the screening, diagnostics, and therapy response monitoring for cancers and other diseases. This approach to studying the kinetics of nanoparticle self-assembly may also provide a valuable tool for understanding the fundamental characteristics of nanoscale particle aggregation.
\end{abstract}

\section{KEYWORDS}

Quantum dot, self-assembly, kinetics, proteomics, diagnostics

\section{Introduction}

Directed self-assembly of nanostructures into microstructures through intermolecular interactions is an important phenomenon in many biological systems. Assembly of virus coat proteins into capsids [1], of microtubulin into microtubules [2], and of collagen [3] and fibrinogen into their respective fibrils are just a few examples where self-assembly plays a critical role in biological processes. Programmed self-assembly using biomolecular interactions as a route to synthesis of novel nanostructured materials has also been an area of active investigation in the recent past $[4,5]$. Techniques aimed at molecular diagnostics have also been demonstrated using nanoparticle self-assembly mediated by molecular interactions, including polynucleotide interactions [6] and antibody-antigen interactions [7].

In a recent publication [8], we demonstrated a novel technique for sub-picomolar quantitative detection of proteomic antigens using single step fluid phase incubation. In this technique, quantum dots (QDs) conjugated with polyclonal antibodies $(\mathrm{Ab})$ through the streptavidin-biotin interaction [9]

Address correspondence to todd.giorgio@vanderbilt.edu 
are incubated with specific antigens in a physiological buffer. The molecular recognition between the antibodies and antigens causes aggregation of the nanoscale conjugates and proteins, resulting in the formation of microscale structures. These larger structures can be easily distinguished from the individual components based on differences in light scattering properties and by other analytical techniques. By sequentially characterizing very small volumes of the reaction mixture, such as via flow cytometry, the microscale aggregates can be quantified. At equilibrium, the antigen concentration and the fraction of events that are classified as aggregates are correlated through a log-linear relationship. Using multiple QD populations with distinct fluorescence emissions, multiplexed detection of two antigens is also feasible (unpublished data) and can, presumably, be extended through the use of alternately biofunctionalized QD with additional fluorescence emission characteristics.

In this short communication, we describe the kinetics of $\mathrm{QD}-\mathrm{Ab}$ aggregation mediated by angiopoietin-2. The kinetics of self-assembly of bio-macromolecules has been studied for many systems, including virus capsid assembly [1, 10], microtubule formation [2, 11, 12], fibril assembly [3], and other protein aggregation phenomena [1316]. Theoretical [17-22] and computational [23-25] studies on these systems illuminate the mechanisms that drive the self-assembly processes. Previous work has also examined factors that influence the kinetics, such as the concentration of various moieties, agitation, and presence of agents that promote or inhibit intermolecular interaction. These factors may be utilized for promotion or inhibition of aggregation [1, 14, 26, 27]. Understanding the kinetics of the present system may improve the specificity and sensitivity of the proposed diagnostic method. Similar studies yielded improvements to the conventional surface-based enzyme linked immunosorbent assay (ELISA), and informed the development of the kinetic ELISA method [28]. While the current study explores the kinetics of highly specific molecular interaction mediated self-assembly, nanoscale material aggregation is important in a wide variety of applications. Controlled aggregation and/or prevention of non-specific aggregation are important considerations for various technologies that use nanomaterials, regardless of the presence or absence of molecular recognition. The techniques developed in this study may provide a novel method of quantitatively characterizing important aspects of nanoscale phenomena.

\section{Experimental}

Streptavidin-coated QDs with $705 \mathrm{~nm}$ fluorescence emission (QD705, \#Q10161MP) were purchased from Invitrogen (Carlsbad, CA) and used as received. Biotin conjugated anti-angiopoietin-2 polyclonal antibody (aA2, \#BAF623), and recombinant human angiopoietin-2 (ang2, \#623-AN) were purchased from R\&D Systems (Minneapolis, MN), reconstituted in Tris-buffered saline (TBS) containing $0.1 \%$ bovine serum albumin (BSA) and stored at $-20^{\circ} \mathrm{C}$. Appropriate dilutions of all antibodies and antigens were prepared in phosphate buffered saline (PBS) with $0.1 \%$ BSA immediately prior to use. Deionized water with resistance of $18 \mathrm{M} \Omega / \mathrm{cm}$ was used for preparing buffers. All buffers were filtered through $0.2 \mu \mathrm{m}$ filters. All other reagents were ACS reagent grade. Flow cytometric measurements were carried out with an unmodified Becton Dickinson (BD) FACSCalibur, and the associated software.

Quantum dot-streptavidin conjugates (QD) and biotinylated anti-angiopoietin-2 polyclonal antibody (aA2) were mixed in PBS-BSA at QD:antibody molar ratio of $1: 3$ and $1 \mathrm{nmol} / \mathrm{L}$ QD concentration. The conjugate was incubated for $30 \mathrm{~min}$ at room temperature and then used as synthesized. The stoichiometry in this protocol has been previously optimized to obviate the need to separate unreacted antibodies from the $\mathrm{QD}-\mathrm{Ab}$ conjugates [8]. The QDaA2 conjugate solution and the ang 2 or control solution at the appropriate dilutions and volumes were added to PBS-BSA for a final volume of 500 $\mu \mathrm{L}$. BSA, similar to ang2 in terms of molecular weight, was used as a negative control for ang2. The reaction mixtures were incubated at room temperature and analyzed by flow cytometry at 5 min intervals from $t=5 \mathrm{~min}$ to $t=90 \mathrm{~min}$, as well as

\section{量 Springer}


immediately after sample preparation, nominally $t=1$ min. Thus, each experiment consisted of nineteen different samples, one for each time point. The influence of ang2 concentration on aggregation kinetics was characterized by carrying out the aggregation reaction with $1 \mathrm{pmol} / \mathrm{L}, 10 \mathrm{pmol} / \mathrm{L}$, and $100 \mathrm{pmol} / \mathrm{L}$ ang2. Control measurements were made at the same time points on samples identical except for the presence of ang2. To compensate for the effect of the variability in the QD-aA2 dilution on the fraction of agglomerates observed, only the datasets with base QD-aA2 count within $2000 \pm$ 200 were utilized for analysis. Three such datasets for each antigen concentration constituted the analyzed data set.

The size, fluorescence, and number of the aggregates in the incubated samples were characterized by flow cytometry. The basic flow cytometric protocol followed was similar to the one used in our previous publication [8]. Briefly, signal amplification parameters for the flow cytometer parameters for forward light scatter (FSC) and side light scatter (SSC) detectors and fluorescence detector with $650 \mathrm{~nm}$ long pass filters (FL3) were optimized for characterizing small particles. The FSC and SSC detector performance was calibrated by using 0.2 , $0.5,1.0,2.0$, and $2.8 \mu \mathrm{m}$ latex calibration particles. In Fig. 1, the regions indicated by R1, R2, R3, R4, and R7 correspond to the $0.2,0.5,1.0,2.0$, and 2.8 $\mu \mathrm{m}$ latex calibration particles, respectively. The data were acquired at low flow rate $(12 \mu \mathrm{L} / \mathrm{min} \pm 3 \mu \mathrm{L} /$ min) for one minute. The FL3 channel was used as the event trigger consistent with the fluorescence emission characteristics of the $705 \mathrm{~nm}$ QDs used in this study. The fraction of aggregates was defined as the event fraction appearing in the upper right quadrant (UR) of the FSC vs SSC plot. FSC greater than 10 a.u. corresponds to a nominal size greater than approximately $0.5 \mu \mathrm{m}$, and results in the observed differentiation of the individual QD-Ab conjugates from the large aggregates inherent in the agglomeration process and the measurement protocol [8]. This fraction was calculated automatically by the CellQuest Pro software. Analysis of the datasets was carried out using the SigmaPlot 9.01 and SigmaStat 3.1 software.

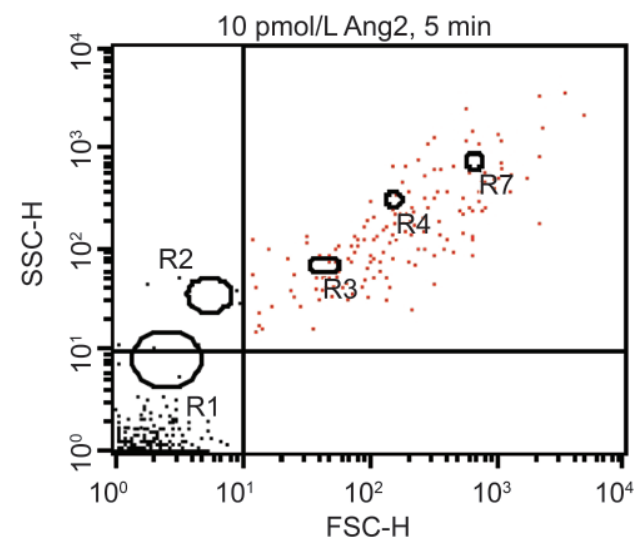

(a)

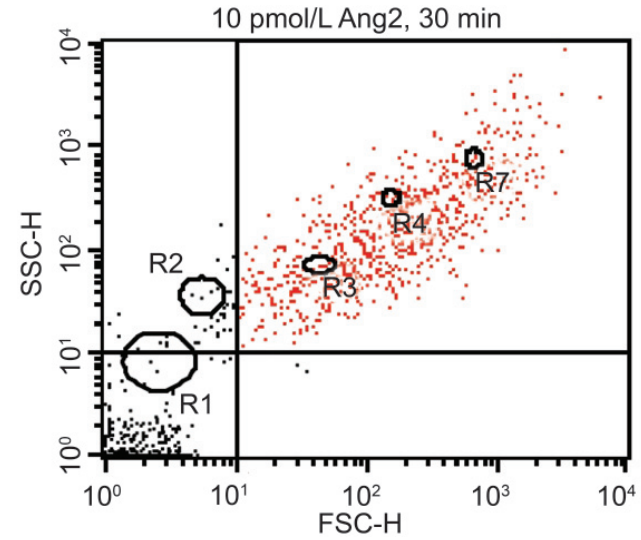

(b)

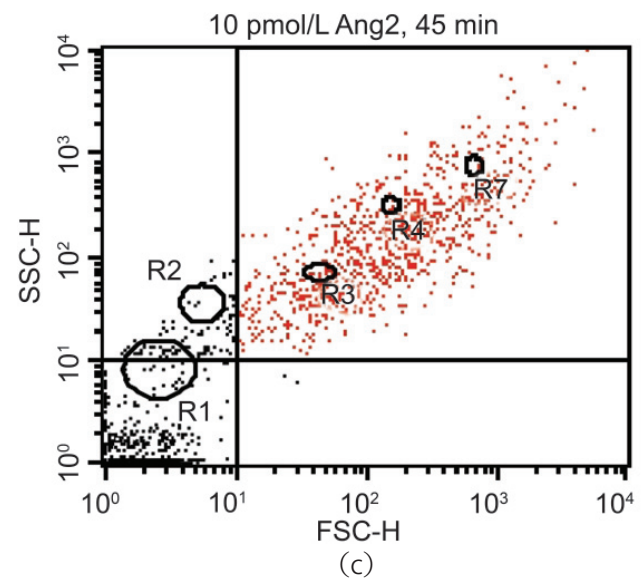

Figure 1 Aggregation kinetics characterized by flow cytometric analysis of ang2 mediated QD-aA2 aggregation at specific time points. Aggregates are quantified as a fraction of total events. (a), (b), and (c) show the raw flow cytometric data depicting increasing aggregate formation with time. These data were acquired for samples with 10 pmol/L QD-aA2 and 10 pmol/L ang2 at 5, 30, and 45 min in (a), (b), and (c), respectively. Each dot represents one particle or 'event' detected. Forward light scatter height (FSC-H) and side light scatter height (SSC-H) intensities are positively correlated with the size and complexity of the particles. Optimization of the detection parameters enables the resolution of micron scale aggregates (red dots, upper right quadrant) from the individual QD-aA2 conjugates and small aggregates (black dots, bottom left quadrant). The ovals labeled R1, R2, R3, R4, and R7 indicate $0.2,0.5,1.0,2.0$, and $2.8 \mu \mathrm{m}$ calibration particles, respectively 


\section{Results and discussion}

The kinetics of ang2 mediated QD-aA2 aggregation exhibits a sigmoidal behavior, with three distinct phases. The initial rate of aggregate formation is slow, most evident from the kinetics for aggregation triggered by the addition of $1 \mathrm{pmol} / \mathrm{L}$ ang2 (Fig. 2). The second phase is characterized by rapid, self-assembled aggregation. The final phase is the reduction of aggregation rate with an asymptotic approach to the equilibrium aggregate fraction. Similar sigmoidal kinetics is observed in many intermolecular interaction-based selfassembly processes $[27,29]$ as well for nanoparticle synthesis [30] and polymer synthesis [29, 31]. Sigmoidal kinetics indicates a thermodynamically unfavorable intermediate in the reaction pathway.

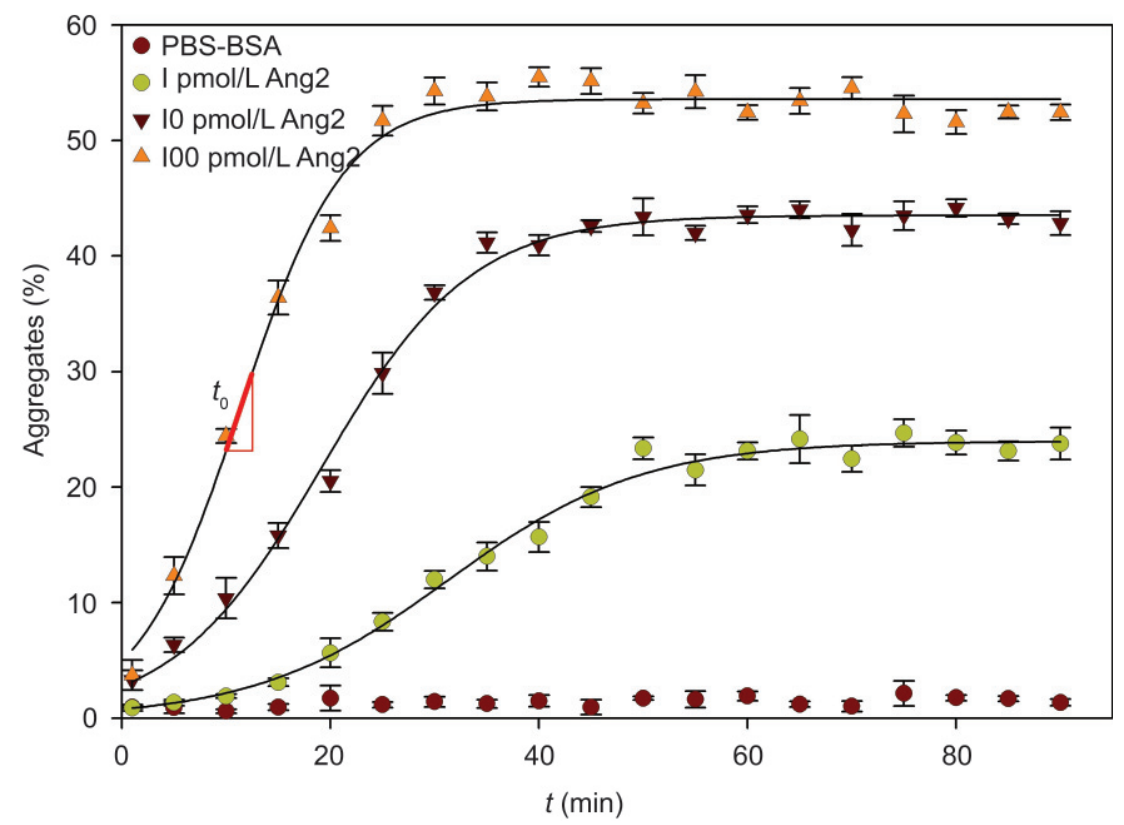

Figure 2 The QD-aA2:ang2 system exhibits a sigmoidal self-assembly kinetics. The parameters of aggregation kinetics in this system are affected by ang2 concentration, as detailed in Table 1. With increasing ang2 concentration, the aggregation rate increases, and time to inflection point $\left(t_{0}\right)$ and time to reach steady state aggregate fraction both decrease. The difference between the slopes, including at $t_{0}$ and at $t=5 \mathrm{~min}$ for the three sigmoid curves suggest the possibility of antigen detection and quantification based on rate of increase in aggregate percent, rather than based on the equilibrium aggregate concentration as described previously [8]. This ability may also be beneficial for detection of molecular biomarkers in complex physiological media, where non-specific intermolecular interactions may have significant effect on the aggregate fraction, especially over long incubation periods. The aggregate fraction is the fraction of total events that are in the upper right quadrant of the SSC vs FSC dot plot (Fig. 1). Each data point in the graph represents the mean and standard deviation of data from three different experiments
The key stages in the aggregation reaction are depicted schematically in Fig. 3, where the first panel represents the QD-Ab conjugates mixed with antigen molecules. Antibody-antigen recognition creates single QD-Ab:antigen complexes, which interact with each other, free QD-Ab conjugates and additional free antigen molecules. This is phase (i) of interactions which leads to the gradual formation of small complexes that act as nuclei for microscale aggregates. (Fig. 3, second panel). During the formation of multi-particle aggregates by molecular recognition, aggregation is favored by the decrease in free energy of the interacting particles, but the entropic cost associated with increased particle organization hinders nucleus formation [32]. Consequently, the probability of formation of micron scale aggregates as well as the aggregation rate are initially both low. Once the nuclei have reached the critical size, the presence of multiple binding sites on the nuclei results in rapid growth of the aggregate [33]. This corresponds to phase (ii). The transition from critical nucleus to large aggregates may occur rapidly. Flow cytometric characterization and dynamic light scattering data published previously [8], show only two particle populationsthe individual, unaggregated QD-aA2:ang2 conjugates and the micron scale aggregates. While the fraction of micron-scale aggregates changes over time, an intermediate population is not apparent in these data. The lack of detectable concentrations of an intermediate is consistent with the data published in the literature for other aggregation systems cited previously. Finally, as the individual components are depleted (Fig. 3, third panel), the aggregation rate slows and the system reaches a stable steady state.

\section{黛 Springer}




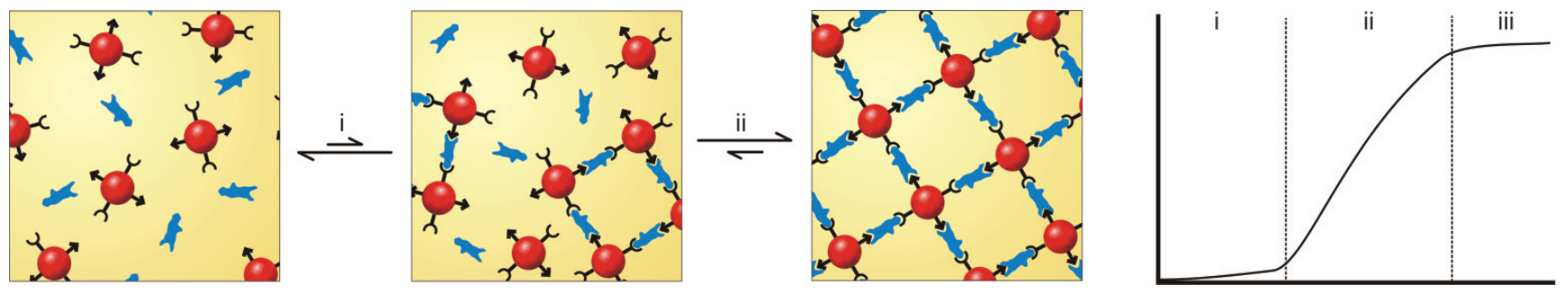

Figure 3 The sigmoidal kinetics observed for QD-aA2:ang2 self-assembly suggests a nucleation limited process, which is schematically depicted here. (i) The entropic cost of particle aggregation may outweigh the reduction in free energy, resulting in slow nucleation; (ii) the presence of multiple binding sites on nuclei of critical size leads to rapid growth of aggregates; (iii) the depletion in available free ang2 causes the asymptotic approach to equilibrium aggregate fraction

Aggregation kinetics observed in the QD-aA2: ang2 system may be described by a three-parameter, sigmoidal curve of the form

$$
a=\frac{a_{\max }}{1-\mathrm{e}^{-\left(\frac{t-t_{0}}{\tau}\right)}}
$$

In Eq. (1), a and $t$ are the variables representing aggregate fraction and time, respectively. The constants for the sigmoids are $a_{\max } t_{0}$, and $\tau$, where $a_{\max }$ is the equilibrium value for $a, t_{0}$ is the time point of inflection, and $\tau$ is a time constant. These parameters are modulated by the ang 2 concentration in contact with the biofunctionalized QDs (Table 1 ). The $a_{\max }$ values obtained from the sigmoidal curves fitted to the $1 \mathrm{pmol} / \mathrm{L}, 10 \mathrm{pmol} / \mathrm{L}$, and 100 $\mathrm{pmol} / \mathrm{L}$ ang 2 datasets are statistically equivalent to the steady state aggregate fraction observed previously [8] for the respective ang2 concentrations. The value of $t_{0}$, which describes the inflection point for the sigmoidal curve, decreases with increasing ang2 concentration. Nucleation-limited aggregation processes, such as the self-assembly described here, accelerate in response to increased concentration of the bridging agent. This behavior, consistently observed in other systems and predicted in many model representations is confirmed in the present work. Increased ang2 concentration results in more rapid attainment of micron sized aggregates from nanoscale QDs, consistent with an increased rate of interactions between the QD-aA2 conjugates and the free ang2 molecules in solution. Thus, increasing ang2 concentration causes earlier attainment of a significant nucleation subpopulation and more rapid transition to the aggregate growth phase. The effect of increasing ang2 concentration on aggregation rate is also evident in the difference in slopes of the aggregation curves. Antigen concentration modulates the rate of aggregate formation at the early time points during the low aggregation rate phase as well as at the inflection point (Table 1). The different slopes observed for different ang2 concentration indicate that an unknown concentration of the target antigen may be estimated from the rate of increase in aggregate count at incubation times far shorter than those required to achieve steady state. The optimal time point for discriminating among antigen concentrations may be determined through comparative analysis of aggregation profiles. The time constant $\tau$ also decreases with increasing antigen concentration and corresponds with the higher rate of aggregation during phase two following nucleations. The ang 2 concentration is correlated with the sigmoid coefficients $a_{\max }, t_{0}$, and $t$, and the slopes at $t=0 \mathrm{~min}$ and $t=t_{0}$, with log linear functions with correlation coefficients 0.96, 0.98, $0.99,0.95$, and 0.99 , respectively. These correlation coefficient values further support the feasibility of quantitatively detecting the biomarker concentration from one or more of the aggregation kinetics parameters. The control samples in the absence of ang2, do not exhibit time dependent aggregation behavior (Fig. 2), further suggesting the potential to use kinetic assessments to detect specific antigens in the presence of other proteins.

\section{Conclusions}

The quantifiable and reproducible self-assembly in the QD-aA2:ang2 system exhibits a sigmoidal kinetic behavior, comparable to that observed in other self-assembly process-based intermolecular 
Table 1 Parameters of the aggregation kinetics sigmoid modulated by antigen concentration

\begin{tabular}{|c|c|c|c|c|c|}
\hline $\begin{array}{l}\text { Ang2 concentration } \\
\text { (picomolar) }\end{array}$ & $a_{\max }(\%)$ & $t_{0}(\min )$ & $T(\min )$ & $\begin{array}{c}\text { Slope } / t=5 \min \\
(\% / \mathrm{min})\end{array}$ & $\begin{array}{c}\text { Slope } / t=t_{0} \\
(\% / \mathrm{min})\end{array}$ \\
\hline 1 & $\begin{array}{c}23.98 \\
+/-0.35\end{array}$ & $\begin{array}{c}31.41 \\
+/-0.38\end{array}$ & $\begin{array}{c}9.27 \\
+/-0.76\end{array}$ & $\begin{array}{c}0.13 \\
+/-0.02\end{array}$ & $\begin{array}{c}0.65 \\
+/-0.05\end{array}$ \\
\hline 10 & $\begin{array}{c}43.51 \\
+/-0.35\end{array}$ & $\begin{array}{c}19.18 \\
+/-0.84\end{array}$ & $\begin{array}{c}7.12 \\
+/-0.16\end{array}$ & $\begin{array}{c}0.65 \\
+/-0.05\end{array}$ & $\begin{array}{c}1.52 \\
+/-0.05\end{array}$ \\
\hline 100 & $\begin{array}{r}53.57 \\
+/-0.30\end{array}$ & $\begin{array}{r}11.35 \\
+/-0.53\end{array}$ & $\begin{array}{c}4.96 \\
+/-0.21\end{array}$ & $\begin{array}{c}1.83 \\
+/-0.09\end{array}$ & $\begin{array}{c}2.68 \\
+/-0.11\end{array}$ \\
\hline
\end{tabular}

Angiopoietin-2 concentration has quantifiable effects on QD-aA2 aggregation kinetics. The fit of sigmoid curves to the aggregation data is described by three constants $a_{\max }, t_{0}$, and $\tau$. The equilibrium aggregate percentage, $a_{\max }$ increases with increasing ang 2 concentration, in agreement with previously published data [8]. The inflection time $t_{0}$, and time constant $\tau$ both decrease with increasing ang2 concentration, indicating faster nucleation and growth of aggregates as a result of higher ang2 concentration. The rates of aggregation, represented by slopes of the sigmoidal curves are also a function of ang 2 concentration, including at $t=5 \mathrm{~min}$, and at $t=t_{0}$. The concentration dependent aggregation rate may enable rapid detection and quantification of target antigens, as well as resolution of specific and non-specific intermolecular interaction. The values represent mean sigmoid coefficients and slopes obtained by fitting a three-parameter sigmoid function to three data-sets individually, and the respective standard deviations. The slopes at $t=5 \mathrm{~min}$ and $t=t_{0}$ were obtained by fitting a linear function to eight sigmoid curve data points centered on the corresponding time points, as generated by SigmaPlot.

interaction. The kinetics of aggregation suggests that the rate of aggregation and the equilibrium extent of aggregation are sensitive to the antigen concentration. Further experimental and theoretical research will be used to characterize the kinetics of multiplexed antigen detection and QD-Ab aggregation in complex biological samples, but is not a part of this short communication. Due to its unique characteristics, this multifunctional nano-conjugate-based analyte detection technique may provide a novel, simple, and rapid mechanism for detecting molecular biomarkers in physiological samples. An understanding of the aggregation kinetics and mechanisms will be particularly important in developing practical implementations of this technology, especially for complex samples containing non-specific antigens with weak intermolecular interactions that may support weak QD-Ab aggregation. Nanoscale particle aggregation in solutions is a fundamental phenomenon that affects various technologies that include nanomaterials. The approach demonstrated here for characterizing nanoparticle aggregation kinetics may be applicable for improving our experimental and theoretical understanding of these nanoparticle interaction mechanisms. Such new fundamental knowledge will presumably be important in fields other than the directed selfassembly and molecular diagnostics applications of the present work.

\section{References}

[1] Zlotnick, A.; Stray, S. J. How does your virus grow? Understanding and interfering with virus assembly. Trends Biotechnol. 2003, 21, 536-542.

[2] Flyvbjerg, H.; Jobs, E.; Leibler, S. Kinetics of selfassembling microtubules: An "inverse problem" in biochemistry. P. Natl. Acad. Sci. USA 1996, 93, 59755979.

[3] Na, G. C.; Butz, L. J.; Carroll, R. J. Mechanism of in vitro collagen fibril assembly. Kinetic and morphological studies. J. Biol. Chem. 1986, 261, 12290-12299.

[4] Whitesides, G. M.; Mathias, J. P.; Seto, C. T. Molecular self-assembly and nanochemistry: A chemical strategy for the synthesis of nanostructures. Science 1991, 254, 1312-1319.

[5] Yan, H.; Park, S. H.; Finkelstein, G.; Reif, J. H.; LaBean, T. H. DNA-templated self-assembly of protein arrays and highly conductive nanowires. Science 2003, 301, 18821884.

[6] Storhoff, J. J.; Elghanian, R.; Mucic, R. C.; Mirkin, C. A.; Letsinger, R. L. One-pot colorimetric differentiation of polynucleotides with single base imperfections using gold nanoparticle probes. J. Am. Chem. Soc. 1998, 120, 1959-1964.

[7] Hirsch, L. R.; Jackson, J. B.; Lee, A.; Halas, N. J. ; West, J. L. A whole blood immunoassay using gold nanoshells. Anal. Chem. 2003, 75, 2377-2381.

[8] Soman, C. P.; Giorgio, T. D. Quantum dot self-assembly 
for protein detection with sub-picomolar sensitivity. Langmuir 2008, 24, 4399-4404.

[9] Goldman, E. R.; Balighian, E. D.; Mattoussi, H.; Kuno, M. K.; Mauro, J. M.; Tran, P. T.; Anderson, G. P. Avidin: A natural bridge for quantum dot-antibody conjugates. J. Am. Chem. Soc. 2002, 124, 6378-6382.

[10] Zlotnick, A.; Aldrich, R.; Johnson, J. M.; Ceres, P.; Young, M. J. Mechanism of capsid assembly for an icosahedral plant virus. Virology 2000, 277, 450-456.

[11] Flyvbjerg, H.; Jobs, E. Microtubule dynamics. II. Kinetics of self-assembly. Phys. Rev. E 1997, 56, 7083-7099.

[12] Bonfils, C.; Bec, N.; Lacroix, B.; Harricane, M. C., Larroque, $C$. Kinetic analysis of tubulin assembly in the presence of the microtubule-associated protein TOGp. J. Biol. Chem. 2007, 282, 5570-5581.

[13] Koo, B. W.; Miranker, A. D. Contribution of the intrinsic disulfide to the assembly mechanism of islet amyloid. Protein Sci. 2005, 14, 231-239.

[14] Sluzky, V.; Tamada, J. A.; Klibanov, A. M.; Langer, R. Kinetics of insulin aggregation in aqueous solutions upon agitation in the presence of hydrophobic surfaces. $P$. Natl. Acad. Sci. USA 1991, 88, 9377-9381.

[15] Speed, M. A.; King, J.; Wang, D. I. C. Polymerization mechanism of polypeptide chain aggregation. Biotechnol. Bioeng. 1997, 54, 333-343.

[16] Spirito, M. D.; Chiappini, R.; Bassi, F. A.; Stasio, E. D.; Giardina, B.; Arcovito, G. Aggregation kinetics and structure of cryoimmunoglobulins clusters. Physica $A$ 2002, 304, 211-219.

[17] Endres, D.; Miyahara, M.; Moisant, P.; Zlotnick, A. A reaction landscape identifies the intermediates critical for self-assembly of virus capsids and other polyhedral structures. Protein Sci. 2005, 14, 1518-1525.

[18] Endres, D.; Zlotnick, A. Model-based analysis of assembly kinetics for virus capsids or other spherical polymers. Biophys. J. 2002, 83, 1217-1230.

[19] Hagan, M. F.; Chandler, D. Dynamic pathways for viral capsid assembly. Biophys. J. 2006, 91, 42-54.

[20] McPherson, A. Micelle formation and crystallization as paradigms for virus assembly. BioEssays 2005, 27, 447458.

[21] Zlotnick, A. Theoretical aspects of virus capsid assembly. J. Mol. Recognit. 2005, 18, 479-490.

[22] Zlotnick, A.; Johnson, J. M.; Wingfield, P. W.; Stahl, S.
J.; Endres, D. A theoretical model successfully identifies features of hepatitis B virus capsid assembly. Biochemistry 1999, 38, 14644-14652.

[23] Bray, D.; Lay, S. Rapid numerical integration algorithm for finding the equilibrium state of a system of coupled binding reactions. Bioinformatics 1994, 10, 471-476.

[24] Bray, D.; Lay, S. Computer-based analysis of the binding steps in protein complex formation. P. Natl. Acad. Sci. USA 1997, 94, 13493-13498.

[25] Lay, S.; Bray, D. A computer program for the analysis of protein complex formation. Bioinformatics 1997, 13, 439-444.

[26] Chong, C. R.; Sullivan, D. J. Inhibition of heme crystal growth by antimalarials and other compounds: Implications for drug discovery. Biochem. Pharmacol. 2003, 66, 2201-2212.

[27] Kodaka, M. Interpretation of concentration-dependence in aggregation kinetics. Biophys. Chem. 2004, 109, 325332.

[28] Hancock, K.; Tsang, V. C. Development and optimization of the FAST-ELISA for detecting antibodies to Schistosoma mansoni. J. Immunol. Meth. 1986, 92, 167 -176 .

[29] Kodaka, M. Requirements for generating sigmoidal time-Course aggregation in nucleation-dependent polymerization model. Biophys. Chem. 2004, 107, 243253.

[30] Towey, T. F.; Khan-Lodhi, A.; Robinson, B. H. Kinetics and mechanism of formation of quantum-sized cadmium sulphide particles in water-aerosol-OT-oil microemulsions. J. Chem. Soc. Faraday Trans. 1990, 86, 3757-3762.

[31] Matsumoto, A.; Kodama, K.; Aota, H.; Capek, I. Kinetics of emulsion crosslinking polymerization and copolymerization of allyl methacrylate. Eur. Polym. J. 1999, 35, 1509-1517.

[32] Sabaté, R.; Gallardo, M. An autocatalytic reaction as a model for the kinetics of the aggregation of $\beta$-amyloid. Biopolymers 2003, 71, 190-195.

[33] Jarrett, J. T.; Lansbury, P. T., Jr. Seeding "one-dimensional crystallization" of amyloid: A pathogenic mechanism in Alzheimer's disease and scrapie? Cell 1993, 73, 10551058. 